\title{
Effect of Particle Size on Fracture Toughness of Polymer Composites
}

\author{
Bernd Lauke \\ Leibniz-Institut für Polymerforschung Dresden e.V., Hohe Str. 6, 01069 Dresden, \\ Germany,e-mail: laukeb@ipfdd.de
}

\begin{abstract}
Fracture toughness of particle reinforced polymers is strongly affected by the size of particles. It can be improved or reduced depending on the materials used and the volume fractions at which the values are compared. Dissipation mechanisms, as particle debonding and subsequent yielding of the polymer, are responsible for the characteristic behaviour. If the debonding energy per volume is considered it can be concluded that smaller particles are favourable for this value. But the product of the specific debonding energy with the dissipation volume is the decisive quantity. Depending on the used debonding criterion, i. e. stress or energy, different conclusions can be drawn. The energy criterion leads to the conclusion that the debonding process inducing fracture toughness independent of particle size, however, the stress criterion at the interface supports the conclusion that smaller particles increase facture toughness.
\end{abstract}

\section{Introduction}

Throughout the last 40 years the subject of improving the mechanical properties of particle filled polymers received large attention and a bulk of publications exists. A survey about this development has been given recently and the reader is referred to [1]. In general, the addition of a hard particle component to a polymer matrix leads to an embrittlement of the resulting composite, if the stress-strain response is considered. It shows a tendency to a reduced necking behaviour with increasing particle volume fraction compared to the bulk matrix. However, if fracture toughness is considered the behaviour is more complex and an increase of fracture toughness with increasing volume fraction of hard particles can be observed. Interfacial debonding determines the initiation and development of the damage process. For glass bead filled epoxies Lee and Yee [2], [3] observed that micro cracking is mainly caused by debonding. However, it was observed that it is limited to a region near the fracture surface and does not occur in a large dissipation zone around the propagating crack. Yielding processes, as diffuse shear yielding and microshear banding, are addressed to be more important for toughening of such composites. Recently Chen et al. [4] examined the influence of particle size on damage dissipation in nanocomposites. They concluded on the basis of an energy debonding criterion that damage dissipation is strongly dependent on the size of particles and that smaller particles are favourable to increase material toughness. But dissipation energy per volume is only one side of the story. The other important point is: how large is the volume in front of the crack where debonding can be initiated, i.e.: Where the stress or 
energy is high enough to debond the particles from the surrounding matrix. A simple geometrical model of particle-particle interaction and a regular particle arrangement is used to consider these points.

\section{Fracture toughness}

To initiate the propagation of an existing crack, energy must be available. The energy release rate $G$ (available from the change of the elastic energy and the applied load for an increment of crack growth) must at least be equal to the energy necessary R (crack resistance) to initiate crack propagation. This is expressed usually as: $G=G_{c}=R(1)$

with $\mathrm{G}_{\mathrm{c}}$ as fracture toughness of the composite (all these quantities as energy per unit area of crack growth). In order to balance the effect of different structural changes on the crack resistance it is necessary to consider the processes near the crack tip in more detail. The fracture processes act in different zones: there are processes immediately near the crack surfaces, which is termed as process zone. The second group are the more extended structural processes, which take place in the so-called dissipation zone. These zones are given in Fig. 1. This kind of subdivision of the region in front of the crack can be traced back to the works by Evans and Faber [5]. The total crack resistance can be calculated by the separate contributions of the mechanisms in the process and dissipation zones, as:

$$
\mathrm{R}=\mathrm{q}_{\mathrm{pz}}+2_{\Sigma \int} \eta_{\mathrm{i}}(\rho) \mathrm{d} \rho \cong \mathrm{q}_{\mathrm{pz}}+2{ }_{\Sigma} \rho_{\mathrm{i}} \eta_{\mathrm{i}}(2)
$$

$\mathrm{i} 0 \mathrm{i}$ where $\mathrm{qpz}_{\mathrm{pz}}$ is the specific fracture energy of the process zone (as energy per unit area of crack), $\eta_{i}$ is the volume density of dissipated energy of the process $(i=1,2, \ldots)$; where the numbers 1 and 2 stand, for example, for particle/matrix debonding $(i=1 \Rightarrow d)$ and matrix yielding processes $(\mathrm{i}=2 \Rightarrow \mathrm{my})$, $\rho$ is the distance

from the crack tip, $\rho_{\mathrm{i}}$ are the dissipation zone radii of the processes (i).

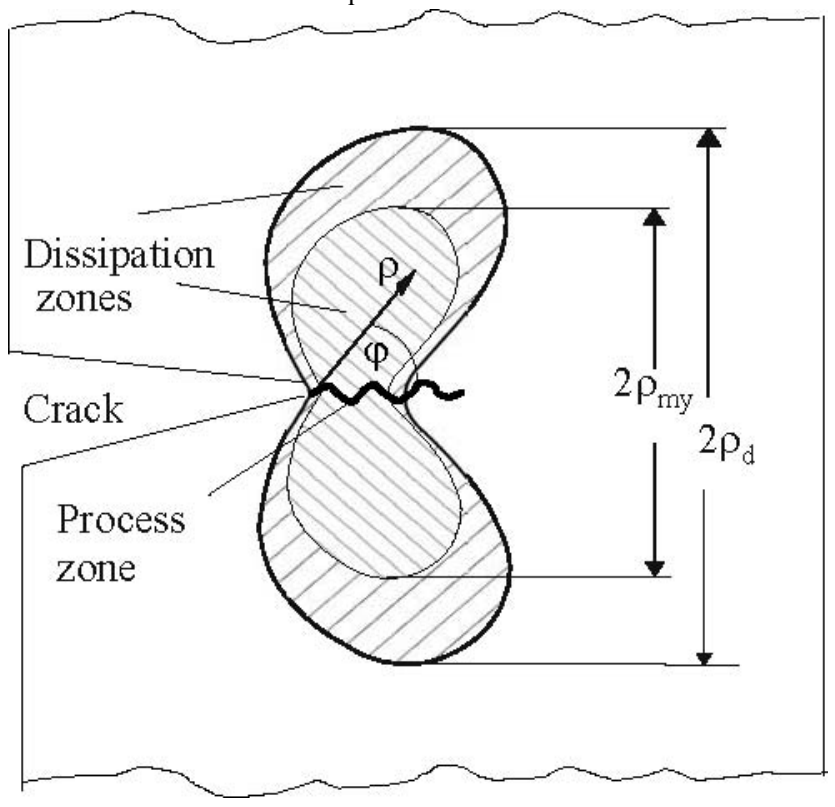


Fig. 1. Dissipation and process zones in front of a crack; radius of debonding zone $\rho_{d}$ and matrix yielding zone, $\rho_{\mathrm{my}}$.

In the summation it is assumed that the different processes do not influence each other directly; indirectly it is considered via reductions of volume fractions, for example during unloading processes caused by debonding. For the calculation of the dissipation radii the approximation for the plastic zone is extended to several energy dissipation mechanisms:

$$
\begin{aligned}
& 1 \mathrm{R} 1 \rho_{\mathrm{i}} \cong \beta \mathrm{RE}_{\mathrm{c}}=\beta(3) \\
& \left(\sigma_{\mathrm{c}}\right)^{2} \mathrm{E}_{\mathrm{c}}\left(\varepsilon_{\mathrm{c}}\right)^{2}
\end{aligned}
$$

where $E_{c}$ is the Young's modulus of the composite, and $\varepsilon_{c}^{i}$ is the local strain in the composite in front of the crack when the material instability (i) initiates, the parameter $\beta$ can be used as a fitting parameter. Inserting Eq. (3) into (2) provides for the crack resistance:

$$
\begin{aligned}
& \mathrm{R}=\stackrel{\mathrm{p} z}{=} \underset{\mathrm{pz}}{\mathrm{q}} \text { (4) } 1-2 \beta \mathrm{E} 1- \\
& \text { c2 } \quad 2^{\Sigma}\left(\sigma^{\mathrm{i}}\right) \mathrm{E}{ }_{\mathrm{c}}^{\Sigma}\left(\varepsilon_{\mathrm{c}}^{\mathrm{i}}\right)
\end{aligned}
$$

\section{Micromechanical model for particle-particle interaction, debonding criterion}

Incorporation of particles into a matrix causes stress concentrations in the neighbourhood of the particle when the composite is under loading. The two-particle arrangement is the typical geometry that should allow describing the main features of the mechanical problem and a simple arrangement was proposed by Evans [6]. The typical situation is shown in Fig. 2; the particles are assumed to have a mean diameter of $d$ and a centre-tocentre distance of $r$ and behave elastically. For the matrix material elastic-plastic material behaviour is assumed.

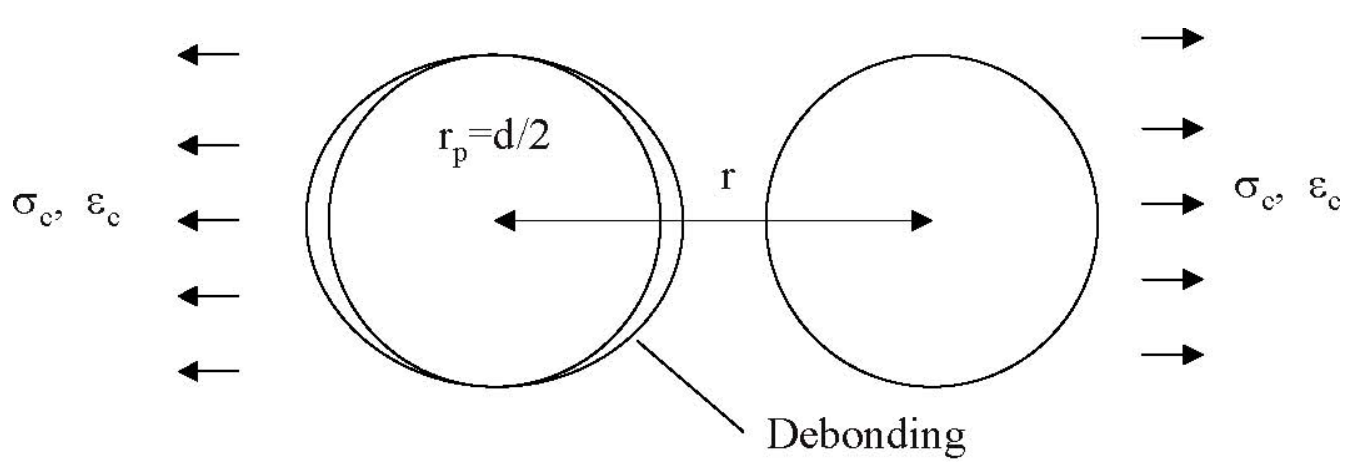

Fig. 2. Representative element for the analytical modelling of local deformations; 
d-particle diameter, r-centre to centre distance, $\sigma_{\mathrm{c}}, \varepsilon_{\mathrm{c}}$-applied stress and strain, respectively, debonding of one particle in a pair.

During loading of this element, the gap (or ligament) between the particles is under maximum deformation that finally reaches a critical value, $\varepsilon_{\mathrm{d}}$, where debonding starts at one of the particles. It is assumed herein that the initiated debonding crack extends all around this particle at this applied load. The debonding leads subsequently to the unloading of the matrix material in the ligament. On the other hand this causes higher loadings in the neighbourhood of the ligament region. The radial, elastic deformation of the matrix in the ligament is approximately given by:

$1 \mathrm{rr} 1$

$$
\begin{gathered}
\varepsilon_{\mathrm{m}}=\sigma_{\mathrm{m}} \approx \varepsilon_{\mathrm{c}}=\varepsilon_{\mathrm{c}}:=\alpha \varepsilon_{\mathrm{c}}=\alpha \sigma_{\mathrm{c}}(5) \\
\mathrm{Er}-\mathrm{dr}-1 \mathrm{E} \\
\mathrm{mc}
\end{gathered}
$$

with the normalization $\mathrm{r}=\mathrm{r} / \mathrm{d}$ and abbreviation $\alpha=$.

$\mathrm{r}-1$ As can be seen from this equation, the strain concentration becomes highest when particles are very close to each other. This leads directly to the critical strain or stress criterion. When the matrix deformation reaches a critical value debonding starts: $\varepsilon_{\mathrm{m}}=: \varepsilon_{\mathrm{d}}$ or $\sigma_{\mathrm{m}}=: \sigma_{\mathrm{d}}(6)$ with $\varepsilon_{\mathrm{d}}$ as the debonding strain or $\sigma_{\mathrm{d}}$ the debonding stress (radial stress at the particle/matrix interface), respectively. Usually the bonding quality in particle reinforced polymers is not very high, so it is assumed herein that debonding starts before matrix yielding: $\sigma_{\mathrm{d}} \leq \sigma_{\mathrm{my}}$ and $\varepsilon_{\mathrm{d}} \leq \varepsilon_{\mathrm{my}}$, with $\sigma_{\mathrm{my}}$ and $\varepsilon_{\mathrm{my}}$ as the matrix yielding stress and strain, respectively. Nicholson [7] considered the problem of particle debonding from a surrounding matrix. He obtained on the basis of an energy consideration (where the deformation energy of the particles is neglected) the following relation of the critical applied stress on a representative element for debonding at the particle/matrix interface:

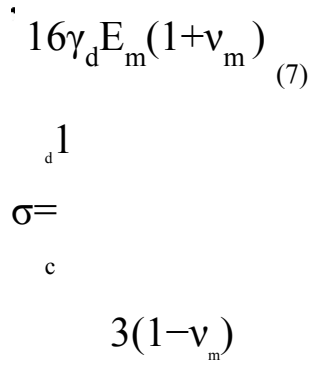

$\mathrm{d}$ with Em and $v_{\mathrm{m}}$ as the matrix modulus and Poisson's ratio, respectively, $2 \gamma_{\mathrm{d}}$, is the specific debonding energy for the two newly created surfaces. For the determination of the debonding stress at the particle surface, the stress 
concentration: $\sigma_{\mathrm{m}}=\mathrm{k} \cdot \sigma_{\mathrm{c}, \mathrm{d}}^{\gamma}$ must be considered. However, it becomes clear that smaller particles demand higher local stresses for debonding to be initiated. Similar results where derived by Chen et al. [4]. The authors considered particle deformation and obtained for the local (radial) debonding stress at the interface:

$\sigma^{\gamma}$

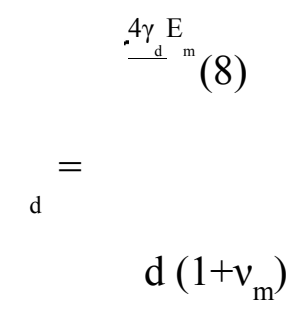

where the "adhesion energies $\mathrm{W}_{\mathrm{a}}$ "of that papers is replaced herein by $2 \gamma_{\mathrm{d}}$.

Thus instead of the debonding stress criterion (6) the following energy criterion

can be used:

$\sigma_{\mathrm{m}}=: \sigma_{\mathrm{d}}^{\gamma}(9)$

The main difference, which has considerable consequences on the structure of the

modelled fracture toughness, is that Eq. (6) provides an experimentally determined value that is of course dependent on the debonding energy but no dependence on the particle diameter was observed until now in experiments. However, Eq. (9) with Eqs. (7) or (8) is explicitly dependent on specific debonding energy, particle diameter and mechanical properties of the polymer. During the production process of particle reinforced polymers the particles are distributed within the matrix. The common aim is to reach a homogeneous distribution without agglomeration of particles. As a first approximation and without loss of relevance for the considered problem, it is assumed that the particles are periodically arranged in a cubic lattice and the strain concentration factor $\alpha$ can be easily calculated. It depends only on the ratio of the interparticle distance to the particle diameter, and this value is related to the particle volume fraction; for this arrangement it is:

$\pi 1 \pi$

$\mathrm{v}=$. Consequently the maximum particle content $\mathrm{v}_{\max }=$ is given for

$6 r^{3} 6 r=1$ where particle are touching each other. Finally, the strain concentration factor $\alpha$ is given by:

$\alpha=1 /\left(1-()^{6 v}\right)$.

$\pi$

\section{Results of fracture toughness modelling}


On the basis of a cubic arrangement it should be possible to explain the typical dissipation processes within the process and dissipation zones. The situations of the dissipation and process zones for static loading is sketchedin Fig. 3 for the case that debonding is initiated before matrix yielding:

,$\varepsilon \leq 1$.
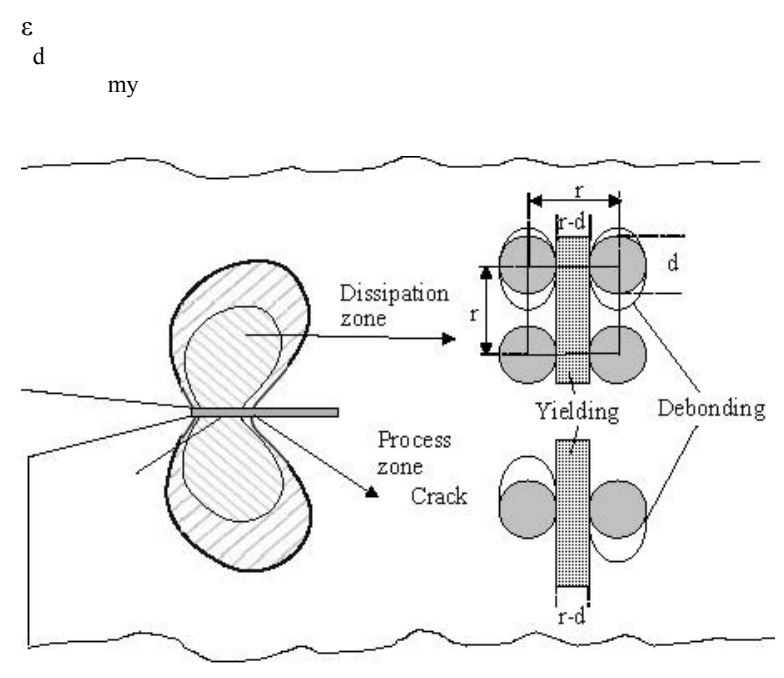

Fig. 3. Debonding and matrix yielding within the process zone (right below) and the same processes within the dissipation zone for a cubic lattice particle arrangement.

Experimental examinations of the fracture process may show that debonding is limited to the very near region around the propagating crack. Then failure is determined by particle debonding in the crack plane and subsequent matrix yielding. In this case the process zone energy of debonding is given by:

$$
\mathrm{q}_{\mathrm{pz}}^{\mathrm{d}}=2 \cdot \gamma_{\mathrm{d}} \cdot{ }_{2}^{\pi \mathrm{d}^{2}} \cdot{ }_{\pi \mathrm{d}^{2}}^{4 \mathrm{v}}=4 \cdot \mathrm{v} \cdot \gamma_{\mathrm{d}}
$$

where the first factor two considers both of the newly created surfaces, the factor two in the denominator considers that only one half of the particle's surface debonds and the last factor is the number of particles per crack plane. Here it is assumed that all particles crossing the crack area are debonded no matter how large (mean diameter) they are. This is reasonable because immediately in front of the crack the elastic stresses are very high, and as assumed debonding starts before yielding. The crack meets already debonded particles, moves around them and fractures the matrix bridges, this leads to the following process zone energy:

my

$\mathrm{q}_{\mathrm{pz}}=2 \cdot \gamma_{\mathrm{m}} \cdot(1-\mathrm{v})$

where it is assumed that the local behaviour equals the crack resistance of the bulk matrix, 
$2 \gamma_{\mathrm{m}}$

Finally, with the basic relation (2), Eq. (12) and $\rho_{\mathrm{d}} \rho_{\mathrm{my}} \approx 0$ the crack resistance is obtained:

$\mathrm{R}=4 \cdot \mathrm{v} \cdot \gamma_{\mathrm{d}}+2 \cdot \gamma_{\mathrm{m}} \cdot(1-\mathrm{v})(13)$ and it is independent on the particle diameter and depends on the specific debonding energy. If, however, debonding takes place in a certain dissipation zone with radius $\rho_{\mathrm{d}}$ then Eq. (4) is the relevant one. To evaluate this equation the critical composite strains $\varepsilon_{c}^{i}$ or stresses $\sigma_{c}^{i}$ must be inserted, and with Eq. (3) this provides:

$$
\begin{gathered}
\mathrm{R}={ }_{2}^{\mathrm{q} z} \text { or } \mathrm{R}={ }_{2}^{\mathrm{p}_{2}}(14) 2 \beta \alpha \eta_{\mathrm{i}} 2 \beta \alpha\left(\mathrm{E}_{\mathrm{m}}\right) \eta_{\mathrm{i}} \\
{ }^{1-} \mathrm{E}_{\mathrm{c}}^{\Sigma}(\varepsilon)^{2} \mathrm{E}_{\mathrm{c}}^{{ }^{2}}(\sigma)^{z}
\end{gathered}
$$

For the determination of the process zone energy in this case it must be considered that debonding causes unloading of some regions around the particles that can not yield, that's why the matrix volume fraction that yields is lower than the matrix volume fraction. From a simple consideration of the geometry, as shown in Fig. 3 it follows:

$$
\mathrm{q}_{\mathrm{pz}}=2 \gamma_{\mathrm{m}} \cdot \quad \mathrm{v}_{\mathrm{my}, \mathrm{pz}}=2 \gamma_{\mathrm{m}} \cdot{ }_{2} \cdot(15) \alpha
$$

Now the debonding and plastic energies must be derived. The volume specific debonding energy, $\eta_{\mathrm{d}}$, is given by dividing the debonding energy of one particle by the representative volume: $2 \pi \mathrm{d}^{2} \gamma_{\mathrm{d}} \pi 16 \mathrm{v}$

$$
\begin{aligned}
\eta_{\mathrm{d}}={ }_{3}{ }_{3} \gamma_{\mathrm{d}}=\gamma_{\mathrm{d}}(16) 2 \mathrm{rdr} \mathrm{d} \text { where it is considered that roughly only half } \\
\text { the number of particles can debond, because, if one particle } \\
\text { debonds the neighbouring one is unloaded and cannot debond. } \\
\text { This equation means that, if all particles have the same (mean) } \\
\text { diameter, } \mathrm{d} \text {, the volume specific dissipation energy will } \\
\text { increase with decreasing particle diameter. The yielding } \\
\text { energy of matrix regions, as shown in Fig. 3, are provided by } \\
\text { some geometrical considerations of their volume relative to } \\
\text { the volume of the representative element, shown on the right } \\
\text { side: } 1 \\
\eta_{\text {my }}=\omega_{\mathrm{m}} \cdot \quad \mathrm{v}_{\mathrm{my}}=\omega_{\mathrm{m}} .
\end{aligned}
$$

$$
\alpha(1-v) \text { with } \omega_{\mathrm{m}} \text { as the volume specific plastic energy of the }
$$

matrix. The determination of that energy is not trivial because it represents the local plastic behaviour of the matrix within a heterogeneous environment and might be quite different from the macroscopic values. However, herein as a first approximation the property of the bulk material is used. Now it is possible to consider the problem of particle size dependence of crack resistance. On the one hand, using the critical stress or strain criterion (Eq. (6)) together with (15), (16) and (17) provides: 8 


$$
\begin{aligned}
& \mathrm{R}={ }^{\alpha^{-2}(\mathrm{v})}(18) 2 \gamma_{\mathrm{m} 2 \beta} \underset{{ }_{\mathrm{d}}^{6 \gamma} \omega \alpha}{ } \mathrm{v}^{2}(\mathrm{v})+{ }_{\left(\varepsilon_{\text {my }}\right)^{2}(1-\mathrm{v})}^{\mathrm{m}(\mathrm{v})} \\
& \mathrm{d}\left(\varepsilon_{\mathrm{d}}\right)^{2}
\end{aligned}
$$

As can be seen, the crack resistance depends on the specific debonding energy, $\gamma_{d}$, and the mean particle diameter, $d$. The debonding strain or stress,

respectively can be determined as well as $\gamma_{d}$ experimentally. On the other hand, using the energy criterion of debonding, Eq. (9) with Eq. (8), together with Eqs. (15-17) provides:

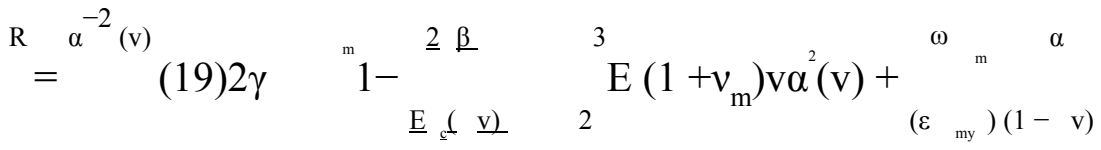

$$
\begin{aligned}
& \text { m2 }
\end{aligned}
$$

Contrary to Eq. (18) the crack resistance now becomes independent of the particle mean diameter, $\mathrm{d}$, and surprisingly also on the adhesion energy. This result is in contradiction to the experimental observations; see $\mathrm{Fu}$ et al [1]. Now the proposed model is applied for glass sphere filled (volume fraction of $\mathrm{v}=0.1$ ) polyethylene with the following material properties of the spheres: elastic modulus $E_{p}=64 \times 10 \mathrm{MPa}$ and Poisson's ratio $v_{\mathrm{p}}=0.2$. The properties of the polyethylene matrix are: $\mathrm{Em}=520 \mathrm{MPa}, \nu_{\mathrm{m}}=0.35$ and $2 \gamma_{\mathrm{m}}=2.8$ $\mathrm{kJ} / \mathrm{m}^{2}$, $\omega_{\mathrm{m}}=47 \mathrm{MPa}$ and $\varepsilon_{\mathrm{my}}=0.28$. The specific debonding energ at the particle $/ \mathrm{matrix}$ interface is: $\gamma_{\mathrm{d}} \approx 10^{-4} \mathrm{~kJ} / \mathrm{m}^{2}$ and the debonding strain is: $\varepsilon_{\mathrm{d}}=0.2$. And for the composite modulus the relation: $\mathrm{E}_{\mathrm{c}}=\mathrm{E}_{\mathrm{m}}{ }_{\underline{2}(\underline{1} \pm \underline{\mathrm{v}})(\underline{7} \pm \underline{\underline{\mathrm{v}}} \underline{\mathrm{v}})(\underline{2} \pm \underline{\mathrm{v}})}$ proposed by Hashin and Strikman [8] is used. Only the particle diameter dependence is considered in the following. The resultsare shown in Fig. 4. 


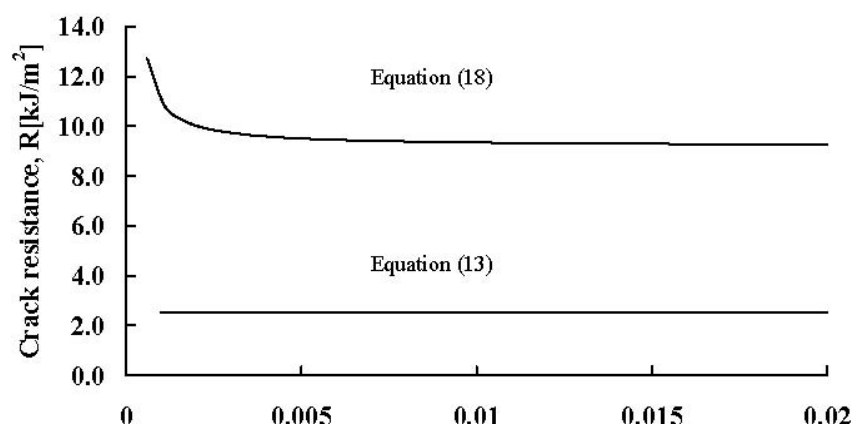

Fig. 4. Crack resistance as a function of particle diameter for the parameters as given within the text.

It reveals that there is a decrease of crack resistance with increasing particle diameter for very small particles. For larger particles the crack resistance remains nearly unaffected by variation of particle diameter because the matrix yielding contribution prevails the energy dissipation. The consideration of debonding and matrix yielding in a dissipation zone provides much higher values than that obtained with the assumption that dissipation takes place only in the process zone (Eq. (13)), cf. Fig. 4.

\section{Discussion and conclusions}

The application of the critical stress criterion leads to a dissipation zone size, which is independent of the particle diameter, as provided by Eqs. (3) and (6). Thus, the increase of crack resistance with decreasing particle size is caused by the increase in the volume specific debonding energy with decreasing particle diameter as reflected by Eq. (16). However, if the energy debonding criterion (Eq. (9)) is applied and inserted into Eq. (3) the dissipation zone radius becomes a function of particle diameter: $\rho_{d} \sim d$, causing the fracture toughness to become independent of the particle

diameter. To improve this simple modelling for a more realistic characterization of composites the following aspects must be taken into account. The volumespecific energy of debonding, $\eta_{d}$, should not be assumed as a constant but the

integral should be carried out in Eq. (2). In real composites the particles are distributed and they are not homogeneous distributed and no less than cubic arranged. Thus a realistic particle distribution function should be considered. And this takes into account that there are pairs of particles, which are very close to each other, causing debonding also for rather low applied stresses as existing away from the crack tip.Further improvement of the modelling can be achieved by consideration of aparticle diameter distribution and by using the elastic-plastic or elastic-viscoplasitc material law of polymers.

\section{References}


[1] S.Y. Fu, X.Q. Feng, B. Lauke and Y.W. Mai, Effects of particle size, particlematrix interface adhesion and particle loading on mechanical properties of particulate polymer composites, Composites Part B 39 (2008) 933-961.

[2] J. Lee and A.F. Yee, Inorganic particle toughening I: micro-mechanical deformations in the fracture of glass bead filled epoxies, Polymer (42) (2001) 577588 .

[3] J. Lee and A.F. Yee, Inorganic particle toughening II: toughening mechanisms of glass bead filled epoxies, Polymer 42 (2001) 589-597.

[4] J.K Chen, Z.P. Huang and J.Zhu, Size effect of particles on the damage dissipation in nanocomposites, Composites Science and Technology 14 (2007) 2990-2996.

[5] A.G. Evans and K. T. Faber, Toughening of Ceramics by Circumferential Microcracking, J. of American Ceramic Soc. 64 (1981) 394-398.

[6] A.G. Evans, S. Williams, and P.W.R. Beaumont, On the toughness of particulate filled polymers, J of Mater Sci 20 (1985) 3668-3674.

[7] D.W. Nicholson, On the Detachment of a Rigid Inclusion from an Elastic Matrix, J. Adhesion 10 (1979) 255-260.

[8] Z. Hashin, S. Shtrikman, On some variational principles in anisotropic and nonhomogeneous elasticity, J. Mech. Phys. Solids 10 (1962) 335-342. 